\title{
Fractional-Order Control of a Nonlinear Time-Delay System: Case Study in Oxygen Regulation in the Heart-Lung Machine
}

\author{
S. J. Sadati, A. Ranjbar Noei, and R. Ghaderi \\ Faculty of Electrical and Computer Engineering, Babol Noshirvani University of Technology, Babol 47148-71167, Iran \\ Correspondence should be addressed to A. Ranjbar Noei, a.ranjbar@nit.ac.ir \\ Received 6 January 2012; Revised 27 April 2012; Accepted 18 May 2012 \\ Academic Editor: Peilin Fu
}

Copyright () 2012 S. J. Sadati et al. This is an open access article distributed under the Creative Commons Attribution License, which permits unrestricted use, distribution, and reproduction in any medium, provided the original work is properly cited.

\begin{abstract}
A fractional-order controller will be proposed to regulate the inlet oxygen into the heart-lung machine. An analytical approach will be explained to satisfy some requirements together with practical implementation of some restrictions for the first time. Primarily a nonlinear single-input single-output (SISO) time-delay model which was obtained previously in the literature is introduced for the oxygen generation process in the heart-lung machine system and we will complete it by adding some new states to control it. Thereafter, the system is linearized using the state feedback linearization approach to find a third-order time-delay dynamics. Consequently classical PID and fractional order $P I^{\lambda} D^{\mu}$ controllers are gained to assess the quality of the proposed technique. A set of optimal parameters of those controllers are achieved through the genetic algorithm optimization procedure through minimizing a cost function. Our design method focuses on minimizing some famous performance criterions such as IAE, ISE, and ITSE. In the genetic algorithm, the controller parameters are chosen as a random population. The best relevant values are achieved by reducing the cost function. A time-domain simulation signifies the performance of $P I^{\lambda} D^{\mu}$ controller with respect to a traditional optimized PID controller.
\end{abstract}

\section{Introduction}

During the complex heart surgery like heart transplantation it may be needed to temporarily interrupt the heart beat. As a result the heart gets cold and set to a resting condition. In this situation the blood, which provides enough oxygen, is not fed to the human body. However this quickly harms the tissues of the body. Even by applying a body-cooling technology (hypothermia), the tissue damage cannot be prevented for a bigger duration time. Thus an artificial machine is essential to take over the functions of the heart and the lung during the surgery. This machine is called heartlung machine (HLM) which will be located in extracorporeal circulation (ECC). The HLM receives the blood from vein, removes the carbon dioxide $\left(\mathrm{CO}_{2}\right)$ from the blood, and adds required oxygen to the blood whilst delivering to the artery. In 1960 the HLM machine was used in the heart surgery for the first time. It is now well improved and still is in use as a reliable and effective method $[1,2]$.

An HLM is connected to the vascular system of the patient body by some specific tubes. During the cardiopulmonary bypass $(\mathrm{CPB})$ surgeries the patient will be anaesthetized. Thus many vital and automatic tasks of the body will be interrupted. There is an alternative oxygenator as an artificial lung in the HLM machine to take over the function of gas exchange through propagation in a thin membrane with a high surface $\left(1.8 \mathrm{~m}^{2}\right)$.

This gas exchange operations will be controlled by the gas fractions and the flow rate of the total mixed gases injected to the oxygenator. This operation is still regulated manually. Nowadays, there is no automatic commercial blood-gas machine. Since the gas exchange operation in the oxygenator is a complicated and nonlinear process with delays together with longtime constants, a manual control of this process is somehow difficult [1-3]. This problem arises due to nonlinear and time-delay phenomena in the heartlung machine. Therefore an automatic approach must be taken in action for the gases regulation in the HLM. This is an auxiliary aim to investigate its performance.

PID controller is the most commonly used controller in industry due to its simple construction and regulation [4-6]. This controller can be a useful tool in the heart-lung machine control. According to the development of fractional order calculus application in recent years, the researchers are trying 
Table 1: Optimum setting of $P I D$ and $P I^{\lambda} D^{\mu}$ controller parameters.

\begin{tabular}{|c|c|c|c|c|c|c|c|}
\hline & $\begin{array}{c}(\mathrm{Z}-\mathrm{N}) \\
k_{c r}=1.12 \times 10^{-5}, T_{c r}=284\end{array}$ & IA & & ISH & & ITA & \\
\hline & PID & PID & $P I^{\lambda} D^{\mu}$ & PID & $P I^{\lambda} D^{\mu}$ & PID & $\mathrm{P} I^{\lambda} D^{\mu}$ \\
\hline$k_{p}$ & $0.6 k_{c r}=6.7 * 10^{-6}$ & $1.44 * 10^{-5}$ & $5.67 * 10^{-5}$ & $1.2692 * 10^{-5}$ & $1.2503 * 10^{-4}$ & $1.2652 * 10^{-5}$ & $6.98 * 10^{-5}$ \\
\hline$k_{i}$ & $1.2 k_{c r} / T_{c r}=5.09 * 10^{-8}$ & $8.43 * 10^{-8}$ & $4.45 * 10^{-8}$ & $1.72 * 10^{-7}$ & $5.6209 * 10^{-6}$ & $6.54 * 10^{-8}$ & $3.0773810^{-8}$ \\
\hline$k_{d}$ & $4.8 k_{c r} / T_{c r}=2.0364 * 10^{-7}$ & 0.00126 & 0.0256 & 0.00233 & 0.08011 & $8.0020 * 10^{-4}$ & 0.03688 \\
\hline$\lambda$ & 1 & 1 & 1.20 & 1 & 0.01 & 1 & 1.25 \\
\hline$\mu$ & 1 & 1 & 1.51 & 1 & 1.77 & 1 & 1.55 \\
\hline
\end{tabular}

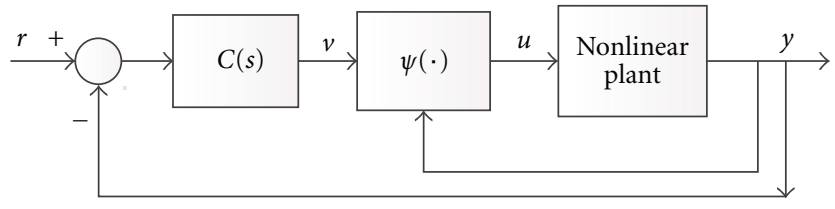

FIGURE 1: A block diagram of the linearization of the HLM control system.

to extend the common PID controller use to the fractional controller [7]. This is almost because a complex model of the controller can be briefly introduced by a concise fractional model. A fractional order PID controller was first introduced by Podlubny $[8,9]$ by defining it as $P I^{\lambda} D^{\mu}$ with $\lambda, \mu \in R$. He has also shown that the stability and the performance characteristics of a system can be improved through choosing a real value for the integral and derivate orders. Frequency domain approach by using fractional order PID controllers was also studied in [10]. Further research activities run in order to define new effective tuning techniques for nonintegerorder controllers by an extension of the classical control theory. To this respect, in [11] the extension of derivation and integration orders from integer to noninteger numbers provides a more flexible tuning strategy and therefore an easier achieving of control requirements with respect to classical controllers. In [12] an optimization method is proposed for tuning of FOPID controllers to achieve some specified desirable behavior of the controlled system such as gain margin and phase margin, high frequency noise rejection, and output disturbances attenuation. In this paper we use time-domain criterion such as integrated absolute error (IAE), integrated square error (ISE), and integrated time absolute error (ITAE). Consequently a fractional order controller is presented whilst the quality of the application in terms of the time response of heart-lung machine is also assessed. In this regard a genetic algorithm is used to find a set of the controller coefficient. This will be done through a fitness function to achieve rise in the performance indices of the rise time, overshoot, and settling time.

The rest of the paper is organized as follows. First a fractional order $P I^{\lambda} D^{\mu}$ controller is introduced. Then a regular model for the heart-lung machine is introduced involving time delays.

In the current literature several efforts have been made in the feedback linearization technique besides analytical

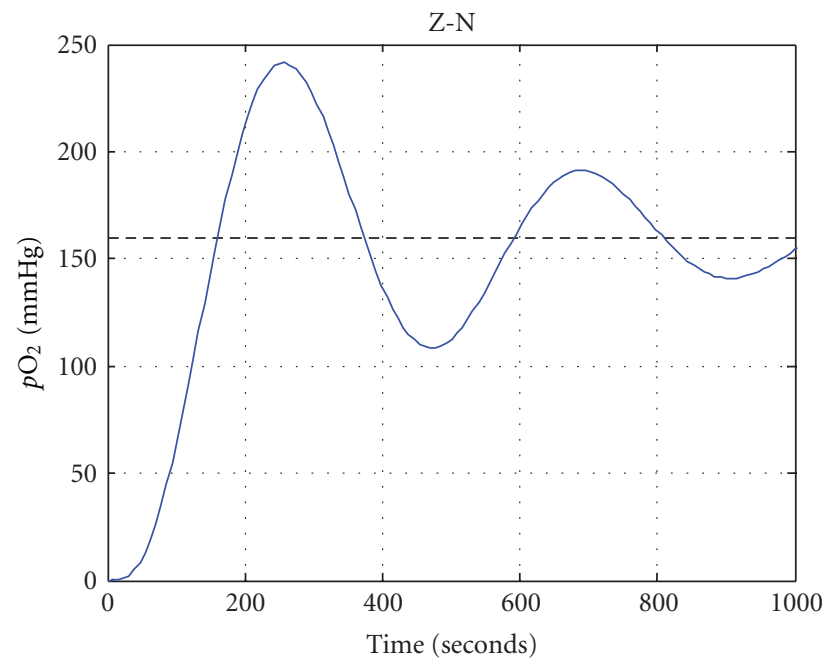

FIgURE 2: Step responses of the oxygenator controlled by PID controller with Z-N tuning rule.

approaches are provided to linearize and design appropriate controller hereafter. Therefore the model is reduced to a linear model by using a state feedback linearization technique. Then classical $P I D$ and fractional $P I^{\lambda} D^{\mu}$ controllers are applied to the linear system. Parameters of the PID are first tuned through Ziegler-Nichols method. Since the obtained parameters result a response with large overshoot together with long settling time, a better output response is of the request. Simultaneously, a best response of using different methods is compared in order to find a better outcome. Accordingly, three different criterions are used to achieve an optimum parameter set. Parameters of $P I D$ and $P I^{\lambda} D^{\mu}$ controllers are achieved using genetic algorithm in order to minimize the cost function.

Both $P I D$ and $P I^{\lambda} D^{\mu}$ controllers, where the gains are optimized by genetic algorithm, are applied on the heartlung machine. The quality of the system response is verified in a simulation study.

\section{Fractional Order $P I^{\lambda} D^{\mu}$ Controller}

A real value of the order of integral and derivative terms provides more flexibility in the controller design to achieve a desired response. This motivates many researchers to 


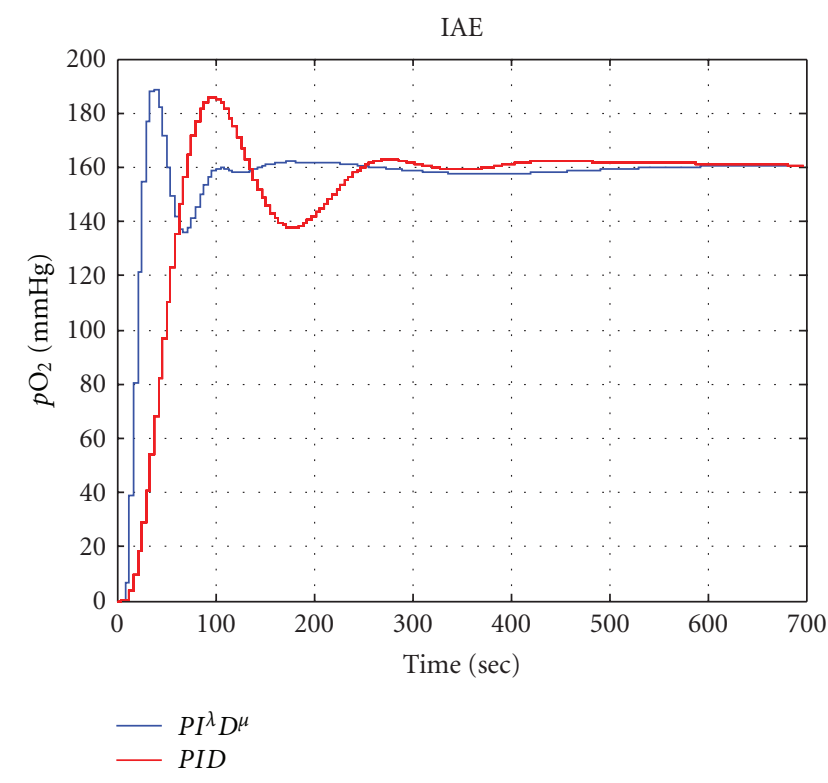

FIGURE 3: Step responses of the oxygenator controlled by PID and $P I^{\lambda} D^{\mu}$ controllers with IAE criteria.

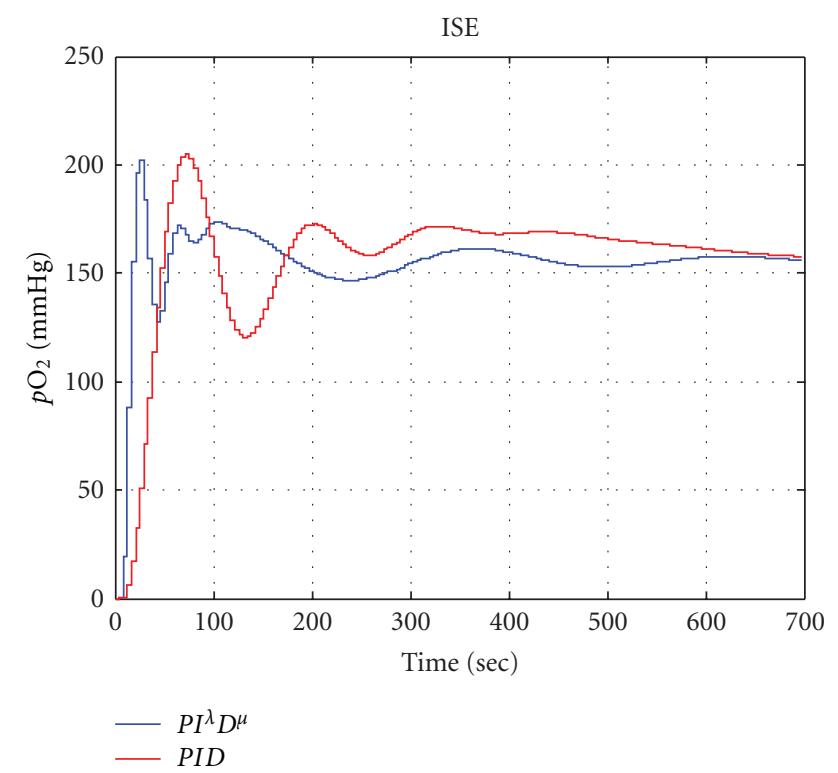

FIgURE 4: Step responses of the oxygenator controlled by PID and $P I^{\lambda} D^{\mu}$ controllers with ISE criteria.

generalize the common controller to the fractional order one [9]. PID controllers are the most widely used controllers in industry. A generalization of classical PID controller to the fractional order one is expressed as $P I^{\lambda} D^{\mu}$ by

$$
C(s)=\frac{U(s)}{E(s)}=k_{p}+\frac{k_{i}}{s^{\lambda}}+k_{d} s^{\mu} \quad \text { with } \lambda, \mu \in \mathbb{R}^{+} .
$$

In fact an appropriate time domain action will be written as follows:

$$
u(t)=k_{p} e(t)+k_{i} D^{-\lambda} e(t)+k_{d} D^{\mu} e(t) .
$$

The performance of $P I D$ and $P I^{\lambda} D^{\mu}$ controllers on the heartlung machine is investigated in the next section.

\section{The Oxygenator Model}

In this paper we will use the proposed model in [2]. However the shortcoming of the previous model will be modified by adding the blood gas analyzer (BGA) dynamics. There is no time-delay seen in the achieved model. Thus it will be convenient for the linearization of the oxygen exchange process model. The linearization process results a system without delay time. Thereafter the produced delay by the mass transportation will be added to the open loop model as a pure delay. Ultimately $P I D$ and $P I^{\lambda} D^{\mu}$ controllers are designed for the time-delay linear system.

The proposed model in [2] explains the gas transportation process in the oxygenator. The model can be considered as a nonlinear single-input-single-output process where the blood flow rate $q_{b}$ may be treated as a predictable perturbation. However from the prior knowledge it is assumed constant at the beginning of the process. Here $\mathrm{p} \hat{\mathrm{CO}} \mathrm{O}_{2}$ is neglected to be considered as a process output. In this paper, just the partial pressure of the blood oxygen is controlled. This system can be described as follows:

$$
\begin{gathered}
\dot{x}=f(x)+b u, \\
y=c x,
\end{gathered}
$$

where $x \in \mathbb{R}^{13}, b \in \mathbb{R}^{13}, u \in \mathbb{R}, y \in \mathbb{R}$, and $f(\cdot)$ is a smooth nonlinear function. Meanwhile

$$
b^{T} u=\left[\begin{array}{lllllllllllll}
0 & 0 & 0 & 0 & 0 & 0 & 0 & 0 & 0 & 0 & 0 & 0 & \frac{q_{g}}{V_{g}}
\end{array}\right] u \text {, }
$$

where $u=\mathrm{pO}_{2, g \text {,in }}=\mathrm{p}_{\mathrm{bar}} \mathrm{FiO}_{2}$ is the control input. Meanwhile, the appeared vascular conditions apart from the blood flow rate $q_{b}$, in the state variables are assumed constant. It is therefore assumed to be a perturbation that directly acts on the states. The state variables are considered as follows:

$$
\begin{aligned}
x & =\left[x_{1}, x_{2}, x_{3}, x_{4}, x_{5}, x_{6}, x_{7}, x_{8}, x_{9}, x_{10}, x_{11}, x_{12}, x_{13}\right]^{T} \\
& =\left[\begin{array}{c}
\mathrm{pO}_{2}, \mathrm{pCO}_{2}, p \mathrm{H}_{\mathrm{virt}},[\mathrm{H}]_{\mathrm{rbc}}, p \mathrm{CO}_{2, \mathrm{rbc}}, \mathrm{pO}_{2}, \\
{\left[\mathrm{HCO}_{3}\right]_{\mathrm{rbc}}, \ldots, p \mathrm{CO}_{2, \mathrm{pl}},[\mathrm{H}]_{\mathrm{pl}},\left[\mathrm{HCO}_{3}\right]_{\mathrm{pl}},} \\
\mathrm{pCO}_{2, g, \text { out }},[\mathrm{carb}], \mathrm{pO}_{2, g, \text { out }}
\end{array}\right]^{T},
\end{aligned}
$$

where $\mathrm{pO}_{2}$ and $\mathrm{p} \hat{\mathrm{CO}}_{2}$ are the measured by BGA values whilst $\mathrm{pH}_{\text {virt }}$ is the corrected value (virtual value) of $\mathrm{pH}$. [H] is the hydrogen ions concentration. $\mathrm{pCO}_{2}$ and $\mathrm{pO}_{2}$ denote the partial pressure of the blood $\mathrm{CO}_{2}$ and $\mathrm{pO}_{2}$, respectively. $\left[\mathrm{HCO}_{3}\right]$ indicates the bicarbonate concentration and [carb] is the carbamate concentration. $\mathrm{pCO}_{2, g, \text { out }}$ and $\mathrm{pO}_{2, g, \text { out }}$ stand for the partial pressure of $\mathrm{CO}_{2}$ and $\mathrm{O}_{2}$ in the gas phase, respectively. "rbc" and "pl" subtitles denote the red blood cells and the blood plasma, respectively. The variables in (5) are replaced with the oxygenator equations. The first 
two equations state the BGA actions in terms of the state representation by

$$
\begin{aligned}
& \dot{x}_{1}=\frac{1}{T_{\mathrm{BGA}}}\left(x_{6}-x_{1}\right), \\
& \dot{x}_{2}=\frac{1}{T_{\mathrm{BGA}}}\left(x_{8}-x_{2}\right) .
\end{aligned}
$$

The values of $\mathrm{pCO}_{2}$ and $\mathrm{pO}_{2}$ processes are considered as output. The equations in [4] are accordingly modified to:

$$
\begin{aligned}
& \dot{x}_{3}=\frac{1}{T_{\mathrm{pH}}}\left[-x_{3}-\log \left(x _ { 4 } \left(\left(0.058 x_{3}-0.437\right) S\left(x_{x}\right)\right.\right.\right. \\
& \left.\left.\left.-0.529 x_{3}+4.6\right)\right)\right] \\
& \dot{x}_{4}=\frac{1}{V_{\mathrm{rbc}}}\left[q_{\mathrm{rbc}}\left([\mathrm{H}]_{\mathrm{rbc}, \mathrm{in}}-x_{4}\right)-V_{\mathrm{rbc}} \frac{2.303}{\beta_{\mathrm{rbc}}} x_{4}\right. \\
& \left.\times\left(-R_{\mathrm{HCO}_{3}, \mathrm{rbc}}+1.5 \dot{x}_{12}-0.6 \operatorname{cap} \dot{S}\left(x_{x}\right)\right)\right], \\
& \dot{x}_{5}=\frac{1}{\alpha_{\mathrm{CO}_{2}} V_{\mathrm{rbc}}}\left[q_{\mathrm{rbc}}\left(\left[\mathrm{CO}_{2}\right]_{\mathrm{rbc}, \mathrm{in}}-\alpha_{\mathrm{CO}_{2}} x_{5}\right)\right. \\
& +D_{\mathrm{CO}_{2, m}}\left(x_{8}-x_{5}\right)+V_{\mathrm{rbc}} R_{\mathrm{HCO}_{3}, \mathrm{rbc}} \\
& \left.-V_{\mathrm{rbc}} \dot{x}_{12}\right] \\
& \dot{x}_{6}=\frac{q_{b}\left(\left[\mathrm{O}_{2}\right]_{b, \text { in }}-\left[\mathrm{O}_{2}\right]_{b}+D_{\mathrm{O}_{2, m}}\left(x_{13}-x_{6}\right)\right)}{V_{b}\left(\alpha_{\mathrm{CO}_{2}}+\operatorname{cap}_{b}\left(d S\left(x_{x}\right) / d x_{x}\right)\left(d x_{x} / d x_{6}\right)\right)}, \\
& \dot{x}_{7}=\frac{1}{V_{\mathrm{rbc}}}\left[q_{\mathrm{rbc}}\left(\left[\mathrm{HCO}_{3}\right]_{\mathrm{rbc}, \text { in }}-x_{7}\right)+D_{\mathrm{HCO}_{3, \mathrm{rbc}}}\left(x_{10}-\frac{x_{7}}{r}\right)\right. \\
& \left.-V_{\mathrm{rbc}} R_{\mathrm{HCO}_{3}, \mathrm{rbc}}\right] \\
& \dot{x}_{8}=\frac{1}{V_{\mathrm{pl}} \alpha_{\mathrm{CO}_{2}}}\left[\begin{array}{l}
q_{\mathrm{pl}}\left(\left[\mathrm{CO}_{2}\right]_{\mathrm{pl}, \mathrm{in}}-\alpha_{\mathrm{CO}_{2}} x_{8}\right) \\
+D_{\mathrm{CO}_{2, m}}\left(x_{11}-x_{8}\right)+D_{\mathrm{CO}_{2, \mathrm{rbc}}}\left(x_{5}-x_{8}\right) \\
+V_{\mathrm{pl}} R_{\mathrm{HCO}_{3, \mathrm{pl}}}
\end{array}\right], \\
& \dot{x}_{9}=\frac{1}{V_{\mathrm{pl}}}\left[q_{\mathrm{pl}}\left([\mathrm{H}]_{\mathrm{pl}, \mathrm{in}}-x_{9}\right)-V_{\mathrm{pl}} \frac{2.303}{\beta_{\mathrm{rbc}}} x_{9} R_{\mathrm{HCO}_{3, \mathrm{pl}}}\right] \text {, } \\
& \dot{x}_{10}=\frac{1}{V_{\mathrm{pl}}}\left[q_{\mathrm{pl}}\left(\left[\mathrm{HCO}_{3}\right]_{\mathrm{pl}, \mathrm{in}}-x_{10}\right)-D_{\mathrm{HCO}_{3, \mathrm{rbc}}}\left(x_{10}-\frac{x_{7}}{r}\right)\right. \\
& \left.-V_{\mathrm{pl}} R_{\mathrm{HCO}_{3, \mathrm{pl}}}\right] \\
& \dot{x}_{11}=\frac{1}{V_{g}}\left(q_{g}\left(\mathrm{p}_{\mathrm{CO}_{2, g, \text { in }}}-x_{11}\right)+D_{\mathrm{CO}_{2, m}} \mathrm{p}_{\mathrm{bar}}\left(x_{8}-x_{11}\right)\right),
\end{aligned}
$$

$$
\begin{gathered}
\dot{x}_{12}=\frac{1}{V_{\mathrm{rbc}}}\left[\begin{array}{c}
q_{\mathrm{rbc}}\left([\mathrm{carb}]_{\mathrm{in}}-x_{12}\right) \\
+k_{a} \alpha_{\mathrm{CO}_{2}} V_{\mathrm{rbc}} x_{5}\left([\mathrm{Hb}]-x_{12}\right) \times\left(\frac{k_{z o} S\left(x_{x}\right)}{k_{z o}+x_{4}}\right. \\
\left.+\frac{k_{z r}\left(1-S\left(x_{x}\right)\right)}{k_{z r}+x_{4}}\right)-V_{\mathrm{rbc}} \frac{k_{a} x_{12} x_{4}}{k_{c}}
\end{array}\right], \\
\dot{x}_{13}=\frac{1}{V_{g}}\left(-q_{g} x_{13}+D_{\mathrm{O}_{2, m}} \mathrm{p}_{\mathrm{bar}}\left(x_{6}-x_{13}\right)\right)
\end{gathered}
$$

A full list of constant values is available in [2]. The carbonic acid hydration is according to (18)-(19) and the oxygen correction curve can be described through (20)-(22):

$$
R_{\mathrm{HCO}_{3}, \mathrm{pl}}=-k_{u} \alpha_{\mathrm{CO}_{2}} x_{8}+\frac{k_{v}}{k} x_{9} x_{10}=f_{\mathrm{HCO}_{3, \mathrm{pl}}}\left(x_{8}, x_{9}, x_{10}\right),
$$

$$
R_{\mathrm{HCO}_{3}, \mathrm{pl}}=\operatorname{cat}\left(-k_{u} \alpha_{\mathrm{CO}_{2}} x_{5}+\frac{k_{v}}{k} x_{4} x_{7}\right)=f_{\mathrm{HCO}_{3, \mathrm{rbc}}}\left(x_{4}, x_{5}, x_{7}\right) \text {, }
$$

$$
\begin{gathered}
{\left[\mathrm{O}_{2}\right]_{b}=\alpha_{\mathrm{O}_{2}} x_{6}+\operatorname{cap}_{b} S\left(x_{x}\right)} \\
p \mathrm{O}_{2, \mathrm{virt}}=x_{x}=x_{6} 10^{0.024\left(37-T_{b}\right)+0.4\left(x_{3}-7.4\right)+0.06 \log \left(40 / x_{5}\right)} \\
S\left(x_{x}\right)=\frac{a_{1} x_{x}+a_{2} x_{x}^{2}+a_{3} x_{x}^{3}+x_{x}^{4}}{a_{4}+a_{5} x_{x}+a_{6} x_{x}^{2}+a_{7} x_{x}^{3}+x_{x}^{4}}
\end{gathered}
$$

where $a_{j}, j=1, \ldots, 7$, are constant parameters obtained from experimental observations. The output vector $c$ in (3) delivers the measured by BGA oxygen partial pressure to the output. Without loss of generality the output is supposed as

$$
y=x_{1}
$$

\section{Time Delay of the HLM System}

The oxygen and neutral gasses mixing process in the mixer consists of a time delay $T_{d_{1}}\left(q_{g}\right)$ [2]. This delay is caused by the metric distance between the mixer and oxygenator due to the gas flow rate in the tubing system. The blood gas analyzer (CDI500, Terumo, Japan) (Terumo Cardiovascular Systems Corporation, 6200 Jackson Road, Ann Arbor, MI 48103/800-262-3304/734-663-4145 (http://www.terumo-cvs.com/)). has an internal sampling time of $T_{s, \mathrm{BGA}}=6 \mathrm{sec}$ with the sample blood $1(\mathrm{mmHg})$. The dominant time constant of $T_{\mathrm{BGA}}$ is shown in (6). The next time delay is concerned with BGA which is indicated by $T_{d_{2}}\left(q_{b}\right)$ for to the blood flow rate. This delay is caused by the blood transportation from the artery, vein, and oxygenator to the BGA sensors. In this case no internal variables of the model are needed. The above two time delays can be integrated as $T_{d}=T_{d_{1}}\left(q_{g}\right)+T_{d_{2}}\left(q_{b}\right)$.

\section{State Feedback linearization}

The model in (6)-(23) for the oxygenator is a nonlinear model with time delay. Excluding the time delay from the 


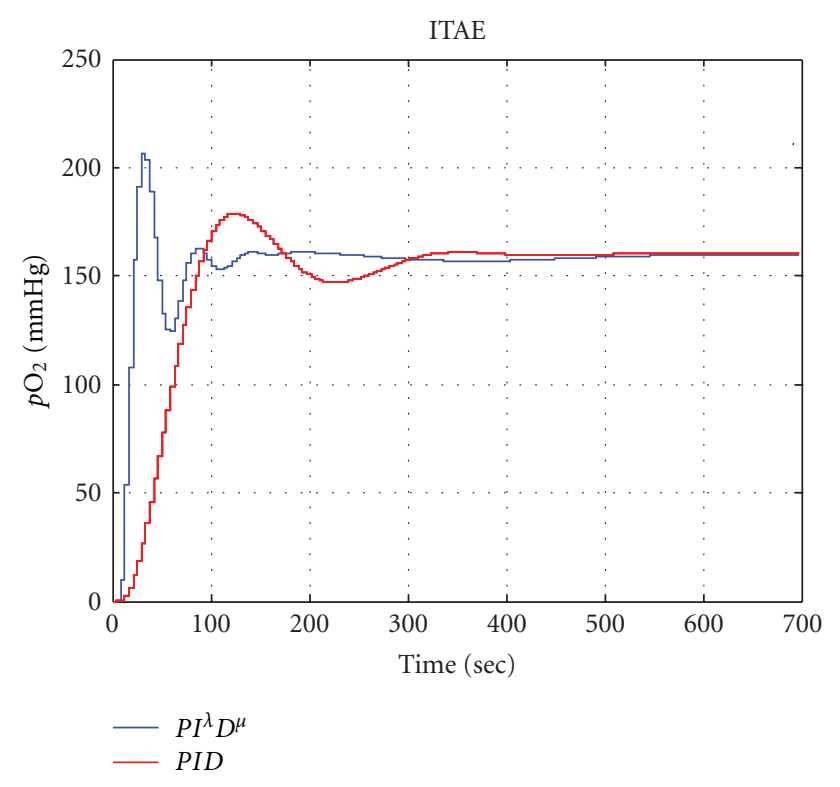

FIGURE 5: Step responses of the oxygenator controlled by PID and $P I^{\lambda} D^{\mu}$ controllers with ITAE criteria.

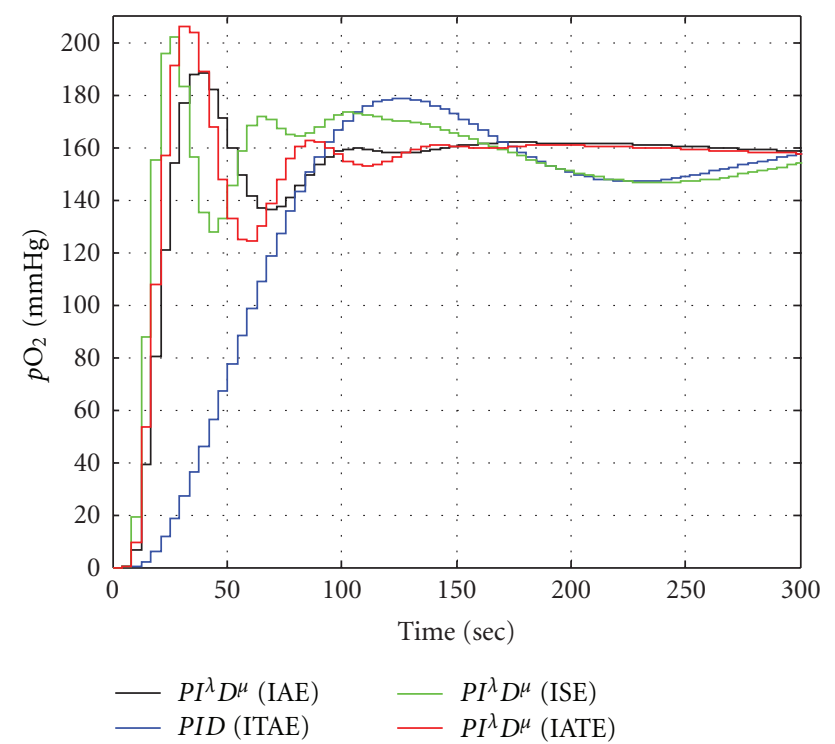

Figure 6: Step responses of the oxygenator with IAE, ISE, and ITAE criteria.

model and by using the state feedback linearization method a linear model is achieved. Later, the time delay is added to the linear model. The linearizing control law using the state feedback is as follows $[13,14]$ :

$$
u=\frac{1}{L_{g} L_{f}^{\gamma-1}}\left(-L_{f}^{\gamma} x_{1}+\nu\right)
$$

where $L_{f} x_{1}$ and $L_{g} L_{f} x_{1}$ denote Lie derivative which is as follows:

$$
\begin{gathered}
L_{f} x_{1}=\frac{\partial x_{1}}{\partial \mathbf{x}} f(\mathbf{x}), \\
L_{g} L_{f} x_{1}=\frac{\partial x_{1}}{\partial \mathbf{x}} g(\mathbf{x}) .
\end{gathered}
$$

In fact the linearization procedure is to continue the output differentiation until the input term " $u$ " appears in the $d^{\gamma} y / d t^{\gamma}$ term such that the coefficient of " $u$ ", that is, $L_{g} L_{f}^{\gamma-1}$ is supposedto be nonzero. Thus, the resultant linear model is of order $\gamma$ with " $\nu$ " as input and " $y$ " as output:

$$
\frac{d^{\gamma} y}{d t^{\gamma}}=v
$$

where $\gamma$ is called the relative degree of the system. If the system in (26) has stable zero dynamics and $\gamma$ as the relative degree of system then the state feedback law is

$$
u=\frac{1}{L_{g} L_{f}^{\gamma-1} x_{1}}\left(-\sum_{j=0}^{\gamma} \beta_{j} L_{f}^{j} x_{1}+\nu\right) .
$$

It stabilizes the system exponentially with the following characteristic polynomial:

$$
s^{\gamma}+\beta_{\gamma-1} s^{\gamma-1}+\cdots+\beta_{1} s+\beta_{0}, \quad \beta_{\gamma}=1 .
$$

The aim is to find the characteristic polynomial which is Hurwitz; that is, the coefficients are positive and the roots are all negative real valued. For the oxygenator system, three times differentiation is needed to make the input appears. Thus the relative degree of the oxygenator system will be found $\gamma=3$. For more details about the state feedback linearization method one may refer to $[13,14]$. However the linear stable system is as follows:

$$
\sum_{j=0}^{\gamma} \beta_{j} y^{(j)}=\nu
$$

In which the parameters $\left(\beta_{0}, \ldots, \beta_{3}\right)$ are chosen such that the input restriction and the sampling time $T_{s, \mathrm{BGA}}=T_{d}=$ $6 \mathrm{~s}$ is met. In other words it cannot be considered a linear system with a very fast response. The "best practice" rule that sample time should be 10 times per process time constant or faster $\left(T_{s} \leq 0.1 T_{\mathrm{p}}\right)$ provides a powerful guideline for setting an upper limit on both control loop sample time and bump test data collection sample time [15]. Therefore poles of linear systems are assumed to be located at $s_{1,2,3}=-0.015$. According to the feedback linearization technique a linear system with time delay is achieved. Consequently the linear transfer function is as follows:

$$
\frac{e^{-6 s}}{(s+0.015)^{3}} .
$$

In this system the total time delay is $T_{d}=6 \mathrm{sec}$. The fraction input oxygen $\mathrm{FiO}_{2}$ and the partial pressure of the oxygen $\mathrm{pO}_{2}$ are considered as the input and output, respectively. 


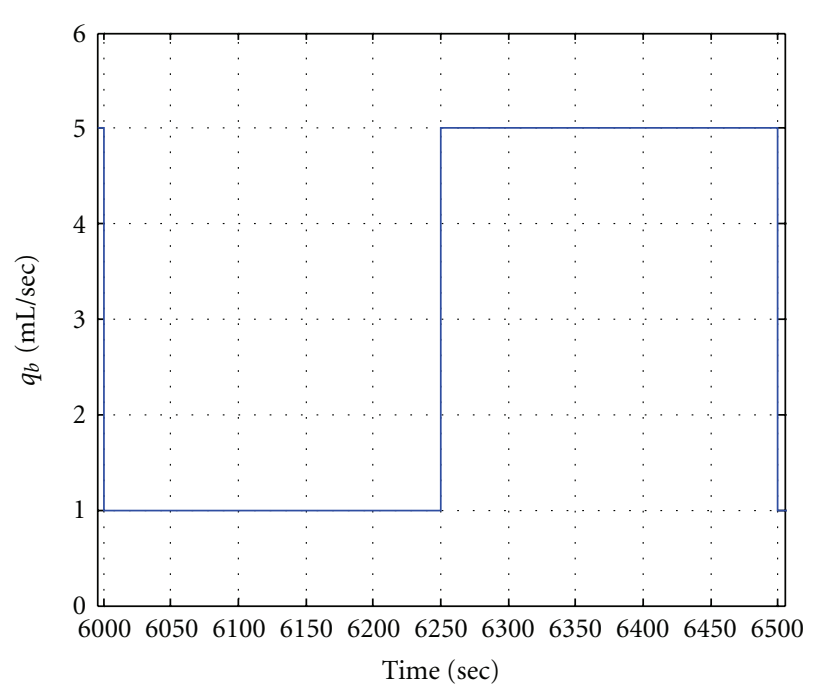

Figure 7: Blood flow as a step disturbance.

\section{The Controller Design and the Simulation}

A state feedback linearization of the oxygenator block diagram of the heart-lung machine is illustrated in Figure 1.

In this paper, classical $P I D$ and fractional $P I^{\lambda} D^{\mu}$ controllers are applied to the oxygenator system. One of the most commonly used methods in tuning of PID parameters is the Ziegler-Nichols method [16]. In this method first the system is set in a closed loop using just with a pure proportional controller. The proportional gain is tuned such that the sinusoidal response is achieved at the output. This gain and the period of the sinusoidal response are treated as the critical gain $\left(k_{\mathrm{cr}}\right)$ and the period $\left(T_{\mathrm{cr}}\right)$, respectively. Then by using Table 1, PID parameters are, respectively, tuned. The appropriate output response using the ZieglerNichols method is stable; however, there are oscillations, overshoot, and rather long settling time at the output response (Figure 2). Thus, an optimal method should have been used in order to obtain a response with fewer settling time with lower overshoot.

Genetic algorithm is one of the optimization methods [17] which is based on the natural selection such as inheritance and mutation. In this algorithm variables which must be optimized are considered as gens. Accordingly set of all variables are defined as a chromosome or a person in the population. Each person or chromosome is considered as a point in the search space. This algorithm needs a cost function or fitness function in order to guide the procedure towards an optimum solution. During the classic PID controller tuning, each of parameters $I, P$, and $D$ is assumed as gen. A set of genes establishes the chromosome or a person. Similarly for the fractional $P I^{\lambda} D^{\mu}$ controller adjustment parameters $P, I, D, \lambda$, and $\mu$ are individually assumed as genes and likewise for the chromosome.

In the genetic algorithm, first an initial population is randomly generated in the search space. Then some persons are randomly selected from the mating pool as parents to breed new children. The crossover operator is applied on

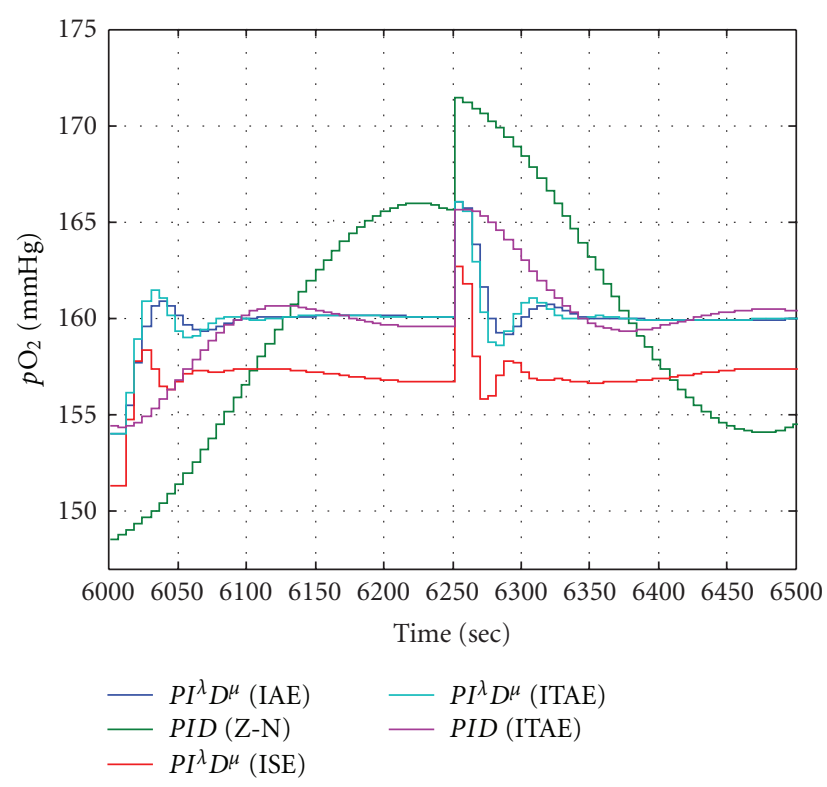

Figure 8: The system responses in presence of a step disturbance controlled by $P I D$ and $P I^{\lambda} D^{\mu}$.

their chromosomes during the breeding procedure. Fitness of each person is calculated according to the fitness function. A person/persons who has/have more fitness is/are considered for the next generation. The genetic algorithm uses the mutation operator in order to escape from local minimum. This operator provides the diversity of the population in the search space for each generation. After some iteration a near optimum solution will be achieved in the search area. In fact three different criterions, IAE, ISE, and ITAE are considered as optimization indexes or the fitness functions in order to find optimum parameters of $P I D$ and $P I^{\lambda} D^{\mu}$ controllers. These are defined as follows:

$$
\begin{gathered}
\mathrm{IAE}=\int_{0}^{T}|e(t)| d t, \quad e(t)=y_{d}(t)-y(t), \\
\mathrm{ISE}=\int_{0}^{T} e^{2}(t) d t \\
\operatorname{ITAE}=\int_{0}^{T} t|e(t)| d t
\end{gathered}
$$

where $y_{d}(t), y(t)$, and $e(t)$ are the desired output (the reference input), the system output, and the error signal respectively. By minimizing these three performance indexes we seek which one of these criterions reduces not only the overshoot but also the steady-state error and the settling time. Finally the genetic algorithm yields optimum parameters which minimize the IAE, ISE, and ITAE indexes according to Table 1.

\section{Simulation Results}

Repeating the genetic algorithm with different initial population achieves an optimum parameters of $P I D$ and $P I^{\lambda} D^{\mu}$ controllers as in Table 1. 
TABLe 2: Indices of the system time response due to using PID and $P I^{\lambda} D^{\mu}$ controllers.

\begin{tabular}{lccccccc}
\hline & $($ Z-N $)$ & \multicolumn{2}{c}{ IAE } & \multicolumn{2}{c}{ ISE } & ITAE \\
& $P I D$ & $P I D$ & $P I^{\lambda} D^{\mu}$ & $P I D$ & $P I^{\lambda} D^{\mu}$ & $P I D$ & $P I^{\lambda} D^{\mu}$ \\
\hline Rise time & $245 \mathrm{sec}$ & $98 \mathrm{sec}$ & $45 \mathrm{sec}$ & $35 \mathrm{sec}$ & $26 \mathrm{sec}$ & $115 \mathrm{sec}$ & $31 \mathrm{sec}$ \\
Settling time & $1783 \mathrm{sec}$ & $300 \mathrm{sec}$ & $100 \mathrm{sec}$ & $612 \mathrm{sec}$ & $565 \mathrm{sec}$ & $300 \mathrm{sec}$ & $115 \mathrm{sec}$ \\
Overshot $\%$ & $50 \%$ & $16.8 \%$ & $18 \%$ & $26.18 \%$ & $26.5 \%$ & $10.12 \%$ & $28 \%$ \\
Reached criteria value & - & 9816.3 & 4626.4 & 769840 & 327900 & 868350 & 465820 \\
\hline
\end{tabular}

\section{Investigation of the Time Indices of the System}

The range of oxygen partial pressure is set to $100-200 \mathrm{mmHg}$ during the surgery. In this paper the oxygen partial pressure is assumed $160 \mathrm{mmHg}$. Thus the reference input is considered as a step function with the amplitude of $160 \mathrm{mmHg}$. Applying the classic and fractional PID controllers with optimum parameters in Table 1 generates the step response of the system as depicted in Figures 2, 3, 4, 5, and 6. To be more specific, the rise time, the settling time, the overshoot, and IAE values are also compared in Table 2. From Figure 2 it can be concluded that despite of the fast response in the Ziegler-Nichols technique there are oscillations and overshoot in the response and the settling time is also high. However, the obtained responses by the GA-tuned PID controllers with IAE, ISE, and ITAE criteria, are found rather better than the classic PID controller counterpart and the Ziegler-Nichols technique. Furthermore the overshoot is found smaller whilst the error is also converged to zero. As shown in Figure 6 the achieved response by the GA-tuned fractional $P I^{\lambda} D^{\mu}$ controller with IAE, ISE, and ITAE results in better response in comparison with the PID controller tuned by GA with IAE, ISE, and ITAE criterias in price of fewer rise and settling time.

\section{Analysis of the Performance of the Controller in Presence of the Perturbation}

The blood flow varies from 1 to $5 \mathrm{~mL} / \mathrm{sec}$ during the surgery. Therefore it is considered as a step disturbance. For analyzing the performance of $P I D$ and $P I^{\lambda} D^{\mu}$ controllers, a step disturbance is applied to the system which is shown in Figure 7. The system response in presence of the disturbance is also depicted in Figure 8 whilst applying PID and $P I^{\lambda} D^{\mu}$ controllers. From Figure 8 it can be seen that the response which is obtained by GA-tuned $P I^{\lambda} D^{\mu}$ controller, converges faster to the steady state with lower overshoot. Additional improvements over the classic PID are also reported in Table 2 .

\section{Conclusion}

In this paper a model of the heart-lung machine with the time delay is considered. The model is treated as a single-input single-output with time delay. The goal in this system is to control the oxygen partial pressure, using the ratio of the input oxygen $\mathrm{FiO}_{2}$. Primarily the system is linearized into a third-order system with time-delay. PID and $P I^{\lambda} D^{\mu}$ controllers are chosen to control the heart-lung machine. The Ziegler-Nichols technique is used to tune PID parameters. Applying this controller results an oscillatory response with large overshoot. Then the GA is used to optimally tune the $P I D$ and $P I^{\lambda} D^{\mu}$ controllers parameters to find a better response with lower overshoot and settling time by minimizing three different criterions IAE, ISE, and ITAE. The time indices are investigated when a step input and disturbance are applied distinctly. Simulation results are presented in Figures 2-8 and Tables 1 and 2. The results verify that $P I^{\lambda} D^{\mu}$ controller, tuned by minimizing IAE criteria in comparison with PID controller which minimized ITAE, provides better performance characteristics in terms of the transient and steady-state response. The outcome is also verified in presence of the step disturbance rejection.

\section{References}

[1] P. H. Kay and C. M. Munsch, Techniques in Extracorporeal Circulation, Arnold, London, UK, 2004.

[2] M. Hexamer and J. Werner, "A mathematical model for the gas transfer in an oxygenator," in Proceedings of the 5th IFAC Symposium on Modelling and Control in Biomedical Systems, Melbourne, Australia, 2003.

[3] J. Allen, A. C. Fisher, J. D. S. Gaylor, and A. R. Razieh, "Development of a digital adaptive control system for $\mathrm{PO}_{2}$ regulation in a membrane oxygenator," Journal of Biomedical Engineering, vol. 14, no. 5, pp. 404-411, 1992.

[4] K. J. Åström and T. Hägglund, "Automatic tuning of simple regulators with specifications on phase and amplitude margins," Automatica, vol. 20, no. 5, pp. 645-651, 1984.

[5] K. J. Åström and T. Hägglund, PID Controllers: Theory, Design and Tuning, Instrument Society of America, North Carolina, USA, 1995.

[6] R. Toscano, "A simple robust PI/PID controller design via numerical optimization approach," Journal of Process Control, vol. 15, no. 1, pp. 81-88, 2005.

[7] C. A. Monje, Y. Chen, B. Vinagre, and V. Feliu, Fractional Order Systems and Controls - Fundamentals and Applications, Springer, 1st edition, 2010.

[8] I. Podlubny, Fractional Differential Equations, Academic Press, San Diego, Calif, USA, 1999.

[9] I. Podlubny, "Fractional-order systems and $P I^{\lambda} D^{\mu}$-controllers," IEEE Transactions on Automatic Control, vol. 44, no. 1, pp. 208-214, 1999.

[10] B. M. Vinagre, C. A. Monje, and A. J. Calderón, "Fractional order systems and fractional order control actions," in Proceedings of the 41st Conference on Decision and Control. Tutorial Workshop 2: Fractional Calculus Applications in Automatic Control and Robotics, Las Vegas, Nev, USA, 2002. 
[11] R. Caponetto, L. Fortuna, and D. Porto, "A new tuning strategy for a non-integer order PID controller," in Proceedings of the 1st IFAC Workshop on Fractional Differentiation and Its Application, pp. 168-173, ENSEIRB, Bordeaux, France, 2004.

[12] C. A. Monje, B. M. Vinagre, V. Feliu, and Y. Chen, "Tuning and auto-tuning of fractional order controllers for industry applications," Control Engineering Practice, vol. 16, no. 7, pp. 798-812, 2008.

[13] S. Sastry, Nonlinear Systems, Springer, New York, NY, USA, 1999.

[14] J. Slotine, Applied Nonlinear Control, Prentice Hall, 1991.

[15] J. Douglas Cooper, http://www.controlguru.com/wp/p35 .html.

[16] J. G. Ziegler and N. B. Nichols, "Optimum settings for automatic controllers," Transactions of the American Society of Mechanical Engineers, vol. 64, pp. 759-768, 1942.

[17] D. E. Goldberg, Genetic Algorithms in Search Optimization and Machine Learning, Addison Wesley, 1st edition, 1989. 

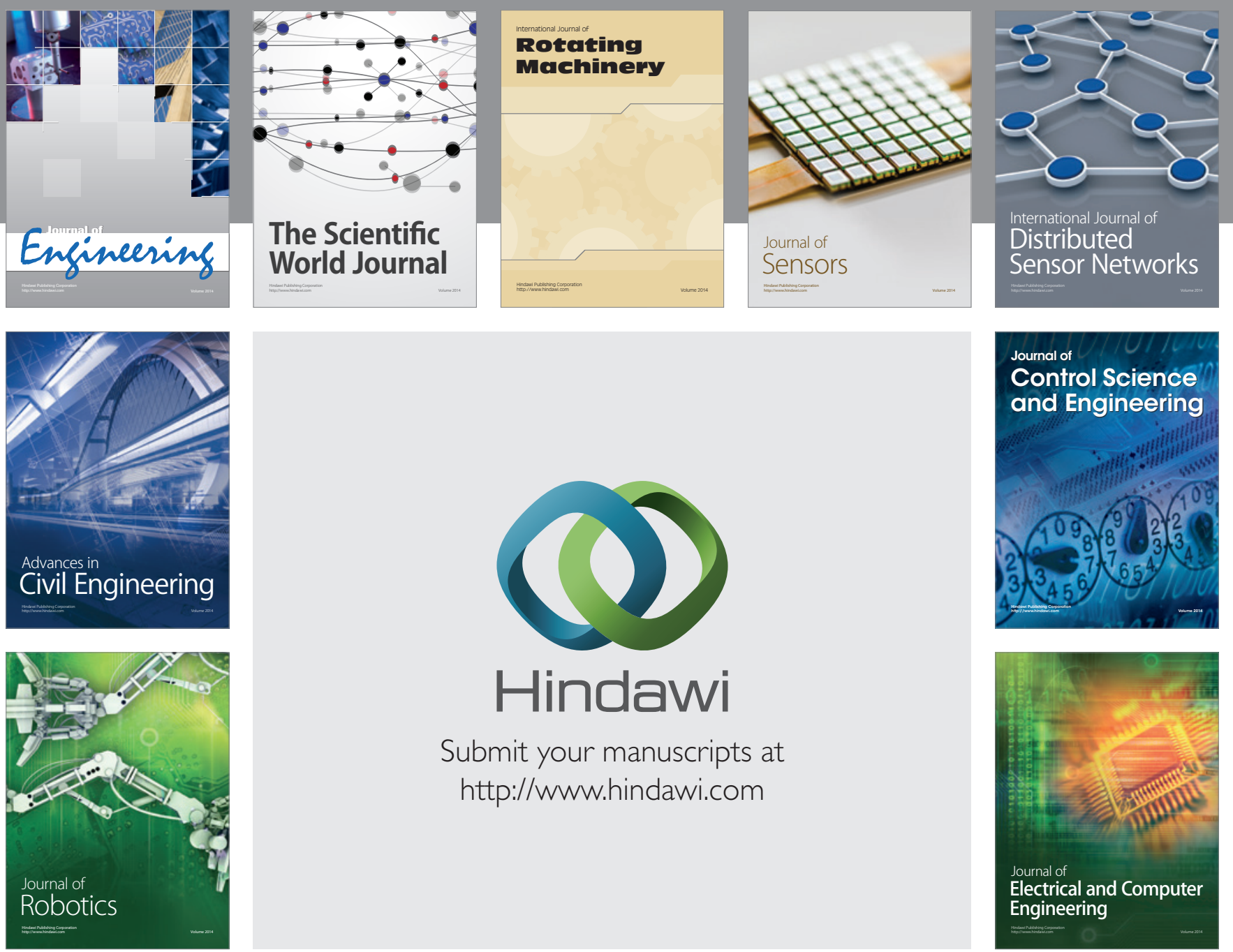

Submit your manuscripts at

http://www.hindawi.com
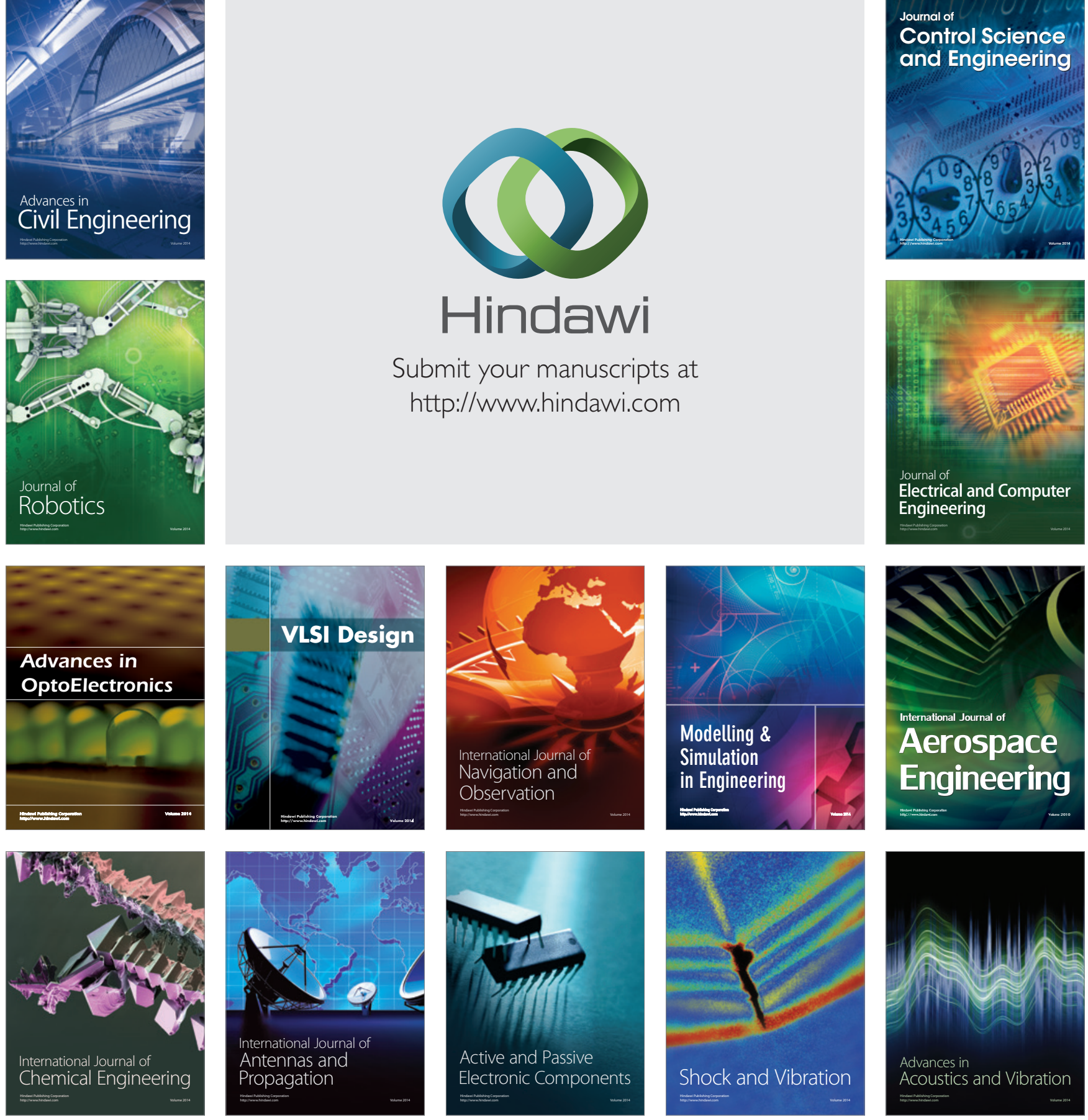